\title{
Extended Standard Hough Transform for Analytical Line Recognition
}

\author{
Abdoulaye SERE, Oumarou SIE \\ Laboratory of Information Processing and Communication \\ University of Ouagadougou \\ Ouagadougou, Burkina Faso \\ Email: abdoulaye.sere@Univ-Ouaga.bf, oumarou.sie@Univ-Ouaga.bf
}

\author{
Eric ANDRES \\ Laboratory XLIM-SIC \\ University of Poitiers \\ Poitiers, France \\ Email: andres@sic.sp2mi.univ-poitiers.fr
}

\begin{abstract}
This paper presents a new method which extends the Standard Hough Transform for the recognition of naive or standard line in a noisy picture. The proposed idea conserves the power of the Standard Hough Transform particularly a limited size of the parameter space and the recognition of vertical lines. The dual of a segment, and the dual of a pixel have been proposed to lead to a new definition of the preimage. Many alternatives of approximation could be established for the sinusoid curves of the dual of a pixel to get new algorithms of line recognition.

Index Terms - hough, transform, recognition, discrete.
\end{abstract}

\section{INTRODUCTION}

In an acquired (photography, satellite picture, scanner) or synthetic picture, we can be interested in different colored areas corresponding to the type of crops, extract the rivers or, in the case of scanner images, determine the contour of organs. To get these information, some manipulations are needed such as the segmentation in region, the recognition and the extraction of the geometric elements such as the border of regions and other kind of processing that may depend on human perception. In image processing, particularly in pattern recognition, we are interested in new extraction and reconstruction techniques in 2D, 3D or higher dimensional objects. Discretization is the transform that associates a discrete object to a continuous one. Reconstruction is the transform that associates a continuous object to a discrete one. There are various ways of considering points in discrete geometry. In this paper, where we focus on dimension two, we consider that a discrete point is represented by a continuous surface element (pixel). One of the question is how to recognize a discrete line.

The Hough Transform, initially defined by Paul Hough in 1962 is very used in lines recognition in a noisy picture. It uses an image space and a parameter space, associates a point in an image space to a line in a parameter space. A lot of methods [3], [4], [5], [6], [7], [8], [9] have been proposed to extend or to improve the Hough Transform in order to permit lines, circles, spheres recognition. An unified definition of the Hough Transform has also been proposed by Maître in [8]. In [5], Martine Dexet introduced the generalized dual space, that extends the Hough Transform to have analytical discrete hyperplane (naive or standard) recognition.

In this paper, we define a new method, which extends the standard hough transform for naive or standard discrete line recognition. The standard Hough transform is based on the polar coordinates of lines. It associates a point in an image space to a sinusoïd curve in a parameter space. The proposed method limits the size of the parameter space.

The starting point of this paper focus on basic notions as the generalized dual space, naive and standard hyperplane definitions and the standard Hough Transform, in the section II. In the section III, we present the extended standard Hough Transform : we define a new dual of a pixel and then the notion of preimage that allows us to establish a recognition algorithm. At the end of this paper, we discuss what still needs to be done in perpectives.

\section{PRELIMINARIES}

In this section, we define some notions to facilitate the understanding of the next sections. These notions concern the generalized dual space, discrete hyperplane definitions and the Standard Hough Transform.

\section{A. Generalized dual space}

In 1962, Paul Hough introduced the Hough Transform. The method uses an image space and a parameter space and is able to recognize objects in noisy pictures. The method has also changed with improvements and extensions to the detection of arcs, circles or sphere.

We define the Hough Transform by :

Definition 1: (Hough Transform) Let $\mathrm{M}\left(X_{0}, Y_{0}\right)$ be a point in an image space $\left(\subset \mathbb{R}^{2}\right)$. The Hough Transform of $M$ is a set of points(a,b) in a parameter space $\left(\subset \mathbb{R}^{2}\right)$ verifying $\mathrm{b}=Y_{0}-\mathrm{a} X_{0}$. Then, the Hough Transform of a point is a line. Let $\mathrm{A}\left(X_{1}\right.$, $\left.Y_{1 a}\right), \mathrm{B}\left(X_{2}, Y_{2}\right), \mathrm{C}\left(X_{3}, Y_{3}\right)$ be three points on an euclidean line defined by the equation $y=a x+b$, where $(a, b) \in \mathbb{R}^{2}$, in an image space. Then, the coordinates of $A, B, C$ verify respectively the equations

$$
\begin{aligned}
& b=Y_{1}-a X_{1} \\
& b=Y_{2}-a X_{2} \\
& b=Y_{3}-a X_{3}
\end{aligned}
$$

The system of equations (1), (2), (3) determines the line parameters $(\mathrm{a}, \mathrm{b})$ that crosses A, B, C. (see the below figure 1) 


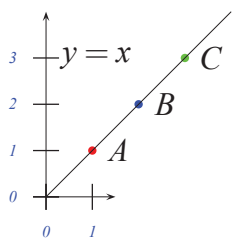

(a) points

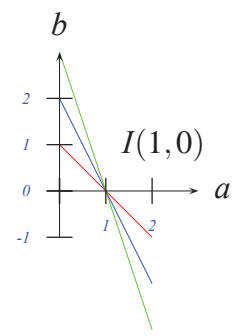

(b) Hough Transform
Figure 1: Hough Transform

As you see in the figure 1 , the coordinates $(1,0)$ of the point I represent the value of the couple $(a, b)$ with $a=1$ and $\mathrm{b}=0$. The line equation correponding to the couple $(\mathrm{a}, \mathrm{b})$ is then obtained by substitution : $\mathrm{y}=\mathrm{ax}+\mathrm{b}$ with $\mathrm{a}=1$ and $\mathrm{b}=0 \Longleftrightarrow$ $\mathrm{y}=\mathrm{x}$. When $\mathrm{a} \rightarrow \infty$, we notice the difficulties to realize vertical lines detection because of the problem to represent an infinity number (a) as a data type that leads to the increasing size of the parameter space. The recognition algorithm consists of the determination of the couples $(\mathrm{a}, \mathrm{b})$ in the parameter space.

The Hough transform according to the definition1 in a continuous space, can not be applied directly on analytical discrete lines recognition because in discrete domain, we work with $\mathbb{Z}^{2}$ points and a discrete line can be seen as a sequence of pixels that are squarre regions centered in $\mathbb{Z}^{2}$ point.

In [5], Martine Dexet extends the Hough transform with the generalized dual space that allows to establish analytical discrete hyperplane (naive or standard) recognition and is equivalent to analytical line recognition in $2 \mathrm{D}$. That leads to build a reconstruction method to obtain a euclidean line from a discrete line .

Definition 2: (The dual of a point in 2D [5]) Let $P_{\xi}\left(x_{1}, x_{2}\right) \in$ $\xi_{2}\left(\subset \mathbb{R}^{2}\right)$ an image space and $P_{P}\left(y_{1}, y_{2}\right) \in P_{2}\left(\subset \mathbb{R}^{2}\right)$ a parameter space be two points. The Dual of $P_{\xi}$ (respectively $P_{P}$ ) is defined by $\operatorname{Dual}\left(P_{\xi}\right)=\left\{\left(y_{1}, y_{2}\right) \in P_{2} \mid y_{2}=x_{2}-x_{1} y_{1}\right\}$ (respectively $\left.\operatorname{Dual}\left(P_{P}\right)=\left\{\left(x_{1}, x_{2}\right) \in P_{2} \mid x_{2}=y_{2}+x_{1} y_{1}\right\}\right)$

As we can see, the definition 2 is equivalent to the Hough transform one and it can be used to establish the dual of an object as follows :

Definition 3: (The dual of an object [6]) Let $O$ be an object of $\xi_{n}\left(\subset \mathbb{R}^{n}\right)$. The Dual of $O$ is defined by Dual $(O)=\bigcup_{p \varepsilon O} \operatorname{Dual}(p)$

We conclude that the dual of a polytope[5] from the definition 3 , particularly the dual of a pixel is the union of the dual of each point.

The preimage[5] is also defined by :

Definition 4: (The preimage of $\mathrm{n}$ pixels [5]) Let $S=\left\{P_{1}, P_{2}, \ldots, P_{n-1}, P_{n}\right\}$ be a set of $n$ pixels $P_{i}$ in $\xi_{2}\left(\subset \mathbb{R}^{2}\right)$ . The preimage of $S$ is defined by $P(S)=\bigcap_{1<i<n} \operatorname{Dual}\left(P_{i}\right)$.

The following figure 2 illustrates the preimage of five pixels.

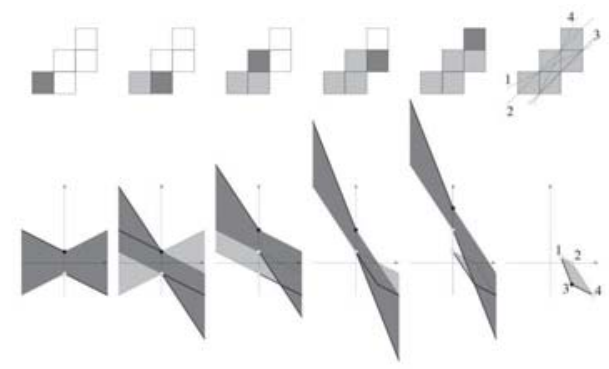

Figure 2: The preimage of five pixels [5]

According to [5], we know that the preimage of $n(n \geq 2)$ pixels give a polygonal area representing a set of points whose coordinates determine the parameters of Naive or Standard lines. As we can see in the figure 2, the preimage of these pixels is a polygon.

If the pixels are vertical, their preimage will be an unlimited polygon [5]; we will need to have a wide size of the parameter space to represent the polygon because the values of the coordinates of the points in the polygon increase, particularly the vertices coordinates. The problem of data types representing these values happens.

One of the question is what is an analytical discrete line in order to build algorithms to recognize them.

\section{B. Analytical hyperplane models}

The analytical hyperplane has been defined by Reveillès [10], [11]. Many models of analytical hyperplanes have been proposed : The bresenham discrete line (Bresenham (1965) Pitteway (1967)), Pythagorean hyperplanes, naive and standard hyperplanes [1], [2].

For this paper, we are interested in naive and standard hyperplane definitions.

Definition 5: (The Naive hyperplane [1], [2]) The Naive hyperplane with the parameters $\left(c_{0}, \ldots, c_{n}\right) \in R^{n+1}$ is the set of points $\left(x_{1}, \ldots, x_{n}\right) \in Z^{n}$ verifying :

$$
-\frac{\max _{1 \leq i \leq n}\left\|c_{i}\right\|}{2} \leq c_{0}+\sum_{i=1}^{n} c_{i} x_{i}<\frac{\max _{1 \leq i \leq n}\left\|c_{i}\right\|}{2}
$$

where $c_{1} \geq 0, c_{1}=0$ and $c_{2} \geq 0$ or $c_{1}=c_{2}=\ldots=c_{n-1}=0$ and $c_{n} \geq 0$.

If $n=2$, we will have the naive line definition from the definition 5 . We obtains a naive line in considering the losange of each pixel, crossed by an euclidean line as we can see in the figure 3 . The losange is also a squarre centered in $\mathbb{Z}^{2}$ point and can be seen as a pixel depending on the choice of the axes of the repere in the image space. 


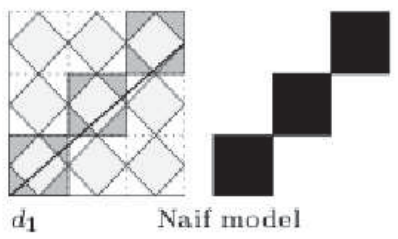

Figure 3: Naive discrete line

Definition : (The Standard hyperplane [1], [2]) The Standard hyperplane with the parameters $\left(c_{0}, \ldots, c_{n}\right) \in R^{n+1}$ is the set of points $\left(x_{1}, \ldots, x_{n}\right) \in Z^{n}$ verifying :

$$
-\frac{\sum_{i=1}^{n}\left\|c_{i}\right\|}{2} \leq c_{0}+\sum_{i=1}^{n} c_{i} x_{i}<\frac{\sum_{i=1}^{n}\left\|c_{i}\right\|}{2}
$$

where $c_{1} \geq 0, c_{1}=0$ and $c_{2} \geq 0$ or $c_{1}=c_{2}=\ldots=c_{n-1}=0$ and $c_{n} \geq 0$.

When $n=2$, we will obtain the standard line definition from the definition 6. We build a standard line in considering the pixels crossed by an euclidean line as we can see in the figure 4.

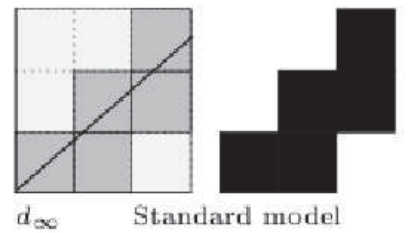

Figure 4: Standard discrete line

Previously in the section II-A, we discuss of vertical line detection problem in the euclidean space. The standard hough transform is a solution to this problem.

\section{Standard ough Transform}

The Standard Hough Transform uses the polar coordinates of lines. It associates a point in a image space to a sinusoïd curve in a parameter space [6].

Definition : Let $\xi$ and be respectively an image space $\left(\subset \mathbb{R}^{2}\right)$ and a parameter space $\left(\subset \mathbb{R}^{2}\right)$ in $2 D$ dimension. Let

$(x, y)$ be a point in $\xi$. The standard ough transform of is the sinusoid curve $S(\quad)$ in defined by $S(\quad)=$ $\left\{(\theta, r) \in \mathbb{R}^{2} / r=x * \cos \theta+y * \sin \theta\right\}$.

The Standard Hough Transform is a sinusoïd curve with the periode $2 \pi$. That means, we can consider $\theta \in[0,2 \pi]$ to analyze the function.

Let $A(1,3)$ be a point in $\mathbb{R}^{2}$. The standard Hough Transform of the point $\mathrm{A}$ is

$$
r=\cos \theta+3 * \sin \theta
$$

According to the definition 7, as we can see in the following figure 5 , the point $\mathrm{A}$ and its dual.
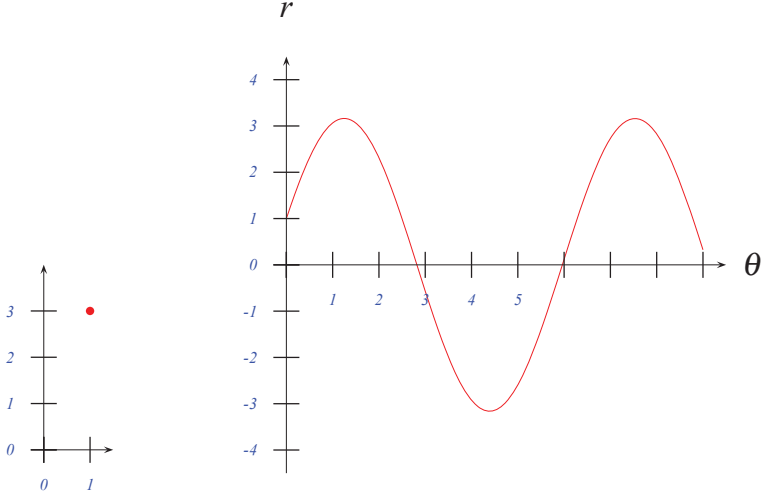

(a) point $(1,3)$

(b) standard hough transform of the point $(1,3)$

Figure 5: Standard hough transform

The recognition algorithm consists to determine the coordinates of the intersection points in the parameter space. In the following figure $6 \mathrm{a}$, we see the points $\mathrm{A}, \mathrm{B}, \mathrm{C}$ on a euclidean line, and in the $5 b$, their dual.

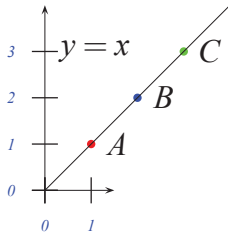

(a) points

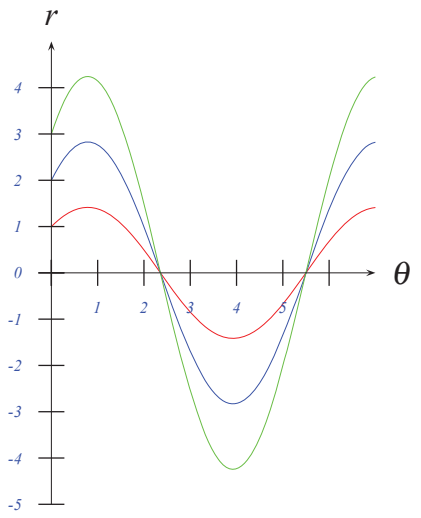

(b) Standard Hough Transform
Figure 6: Standard Hough Transform

If the point $\left(\theta_{1}, r_{1}\right)$ is an intersection point between two sinusoïd curves then the point $\left(\theta_{1}+\pi,-r_{1}\right)$ will be also an intersection point between them because we have :

$$
\begin{array}{r}
r=x * \cos (\theta)+y * \sin (\theta) \Longleftrightarrow-r=x * \cos (\theta+\pi)+y * \\
\sin (\theta+\pi)
\end{array}
$$

Then finally, we can retain $\theta \in[0, \pi]$. That will be useful forward to optimize the computing of the preimage.

The detection of vertical lines is possible, because we can obtain $\mathrm{x}=\alpha$ with $\alpha \in \mathbb{R}$ from $r=x * \cos (\theta)+y * \sin (\theta)$ with $\left(\theta=\frac{\pi}{2}\right.$ modulo $\left.2 \pi\right)$ or $\left(\theta=\frac{3 \pi}{2}\right.$ modulo $\left.2 \pi\right)$.

We have extended the Standard Hough Transform into the discrete aspect. The idea is to conserve the power of the Standard Hough Transform and to have naive or standard line recognition in the proposed method.

\section{EXTENDED STANDARD HOUgH TRANSFORM}

This section highlights the proposed method. Firstly, we will analyse how to compute the dual of a pixel, what can 
be its approximation in order to build the preimage. At the end of this part, we will present an recognition algorithm, the advantages of the proposed method and some illustrations.

We consider here that the term Dual is equivalent to the standard Hough transform.

We conserve the definition 3 of the dual of an object in preliminaries that is the dual of an object $\mathrm{O}$ is the union of the dual of each point of $\mathrm{O}$.

\section{A. Dual of a segment}

The dual of a segment is the union of the dual of each point on this segment. The introduced theorem 1 determines how to compute the dual of a segment.

Theorem 1: The dual of a segment is an area limited by the dual of its extremities

\section{Proof:}

Let us prove that. Let [AC] be a segment in an image space with $\mathrm{C}\left(x_{c}, y_{c}\right)$ and $\mathrm{A}\left(x_{a}, y_{a}\right)$. Let $\mathrm{M}\left(x_{m}, y_{m}\right)$ be a point $\in[\mathrm{AC}]$. We know that the Dual(A), Dual(C), Dual(M) are respectively a set of points $\left(\theta, r_{a}\right),\left(\theta, r_{c}\right),\left(\theta, r_{m}\right)$ verifying respectively the equations :

$$
\begin{aligned}
r_{a} & =x_{a} \cos \theta+y_{a} \sin \theta \\
r_{c} & =x_{c} \cos \theta+y_{c} \sin \theta \\
r_{m} & =x_{m} \cos \theta+y_{m} \sin \theta
\end{aligned}
$$

We have also

$$
\vec{O}=* \overrightarrow{0 A}+(1-) * \overrightarrow{O C}
$$

where $k \in \mathbb{R}, 0 \leq \leq 1$.

We obtain with (6), (7) and (8) that

$$
\text { (9) } \Rightarrow x_{m}=* x_{a}+(1-) * x_{c}
$$

and

$$
\text { (9) } \Rightarrow y_{m}=* y_{a}+(1-) * y_{c}
$$

Then considering (10) and (11) we conclude $r_{m}=*$ $\left(x_{a} \cos \theta+* y_{a} \sin \theta\right)+(1-) *\left(x_{c} \cos \theta+y_{c} \sin \theta\right)$

That implies $r_{m}=* r_{a}+(1-) * r_{c}$.

That means $r_{a} \leq r_{m} \leq r_{c}$ or $r_{c} \leq r_{m} \leq r_{a}$.

So, the curve of Dual(M) is between the curve of Dual(A) and the curve of Dual(C).

Inversely, suppose $\mathrm{N}\left(\theta_{1}, r_{1}\right)$ a point between the curve of $\operatorname{Dual}(\mathrm{A})$ and the curve of Dual(C). We want to show that there exists a point $M \in[A C]$ such as the curve of Dual(M) passes through $\mathrm{N}$.

We have $r_{c}\left(\theta_{1}\right) \leq r_{1} \leq r_{a}\left(\theta_{1}\right)$ or $r_{a}\left(\theta_{1}\right) \leq r_{1} \leq r_{c}\left(\theta_{1}\right)$

That means $\exists \mathrm{k} \in \mathbb{R}$ such as $0 \leq \leq 1$ such as

$$
r_{1}=* r_{a}\left(\theta_{1}\right)+(1-) * r_{c}\left(\theta_{1}\right)
$$

As $r_{a}\left(\theta_{1}\right)=x_{a} \cos \theta_{1}+y_{a} \sin \theta_{1}$ and $r_{c}\left(\theta_{1}\right)=x_{c} \cos \theta_{1}+$ $y_{c} \sin \theta_{1}$

Then, we obtain

$$
\begin{array}{r}
(12) \Rightarrow r_{1}=*\left(x_{a} \cos \theta_{1}+y_{a} \sin \theta_{1}\right)+(1-) * \\
\left(x_{c} \cos \theta_{1}+y_{c} \sin \theta_{1}\right)
\end{array}
$$

So

$$
\begin{array}{r}
(13) \Rightarrow r_{1}=\left(* x_{a}+(1-) * x_{c}\right) \cos \theta_{1}+\left(* y_{a}+\right. \\
\left.(1-) * y_{c}\right) \sin \theta_{1}
\end{array}
$$

Finally (14) implies the point of coordinates $\left(\left(* x_{a}+(1-\right.\right.$ )$\left.\left.* x_{c}\right),\left(* y_{a}+(1-) * y_{c}\right)\right)$ is on the segment [AC].

In the following figure 7 , we see a segment [AC] with $M \in[A C]$ in $7 \mathrm{a}$, in7b, the dual of $\mathrm{M}$ between the dual of $\mathrm{A}$ and $\mathrm{C}$ and in $7 c$ the equivalent surface of the dual of [AC].

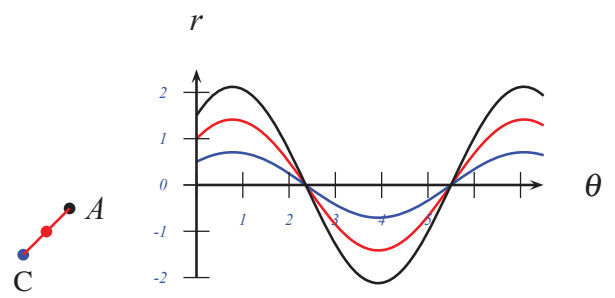

(a) $[\mathrm{AC}]$

(b) Dual of A, M, C

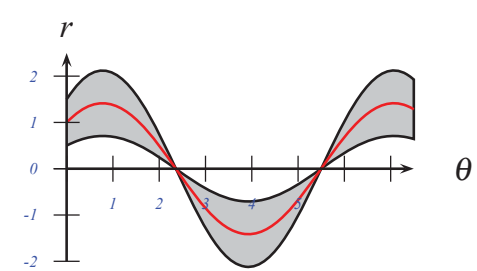

(c) Dual of $[\mathrm{AC}]$

Figure 7: Dual of a segment

\section{B. Dual of a pixel}

A pixel is the unit squarre region centered in $\left(p_{1}, p_{2}\right)$ a $\mathbb{Z}^{2}$ point : $\left\{(x, y) \in \mathbb{R}^{2}|| x-p_{1}\left|\leq \frac{1}{2},\right| y-p_{2} \mid \leq \frac{1}{2}\right\}$. The dual of a pixel is then the union of the dual of each point(x,y). Its definition is :

Definition : Let $p$ be a pixel centered in $\left(p_{1}, p_{2}\right)$ in a image space $\xi$. The Dual of $p$ is a set of points of the parameter space , defined by Dual $(p)=\left\{(\theta, r) \in 2 / \forall(\alpha, \beta) \in\left[\frac{-1}{2}, \frac{1}{2}\right]^{2}, r=\right.$ $\left(p_{1}+\alpha\right) \cos \theta+\left(p_{2}+\beta\right) \sin \theta$ ?

The figure 8 shows the standard Hough transform of vertices of pixel $(1,1)$ : Each colored vertex correspond to its colored curve image.

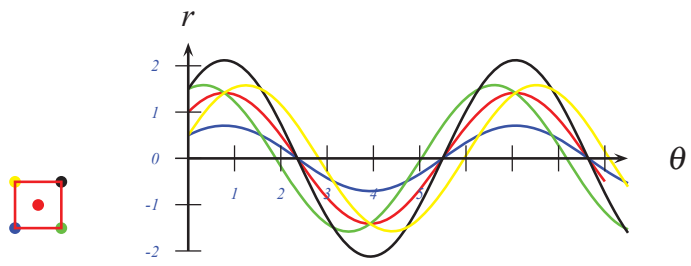

$\begin{array}{ll}\text { (a) } \operatorname{pixel}(1,1) & \text { (b) Standard hough transform of vertices }\end{array}$

Figure 8: Extended standard hough tranform 
Let $\mathrm{A}, \mathrm{B}, \mathrm{C}, \mathrm{D}$ be the vertices of a pixel $\mathrm{p}$ as ilustrated in the figure 9 . A pixel is a set of an infinity of vertical segments (9a) or an infinity of horizontal segments (9b). We have also the diagonal segments in (9c).

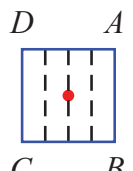

(a)

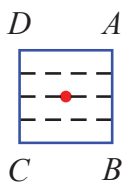

(b)

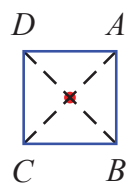

(c)
Figure 9: Sides of a pixel

Our aim is to determine the dual of a pixel in going with the dual of its segments (vertical, diagonal or horizontal).

We propose the following theorem 2 .

Theorem 2: Let $\mathrm{p}$ be a pixel in $\xi_{2}\left(\subset \mathbb{R}^{2}\right)$. Its dual is a area limited by the curve of the duals of its vertical sides. This area is also limited by the curve of the duals of its horizontal sides or by the duals of its diagonals.

Proof: Let us prove that. Let $\mathrm{p}$ be a pixel with its vertices A, B, C, D in image space like in the figure 10 .

Firstly, one shows that the dual of a pixel is an area limited by the dual of its vertical sides $[A B]$ and $[C D]$. The dual of a segment is obtained by the theorem 1 . Let $\mathrm{N}$ be a point in a pixel p with vertices $\mathrm{A}, \mathrm{B}, \mathrm{C}, \mathrm{D}$ where $\mathrm{N} \notin\{A, B, C, D\}$. There exists two points I, J such that $\mathrm{N} \in[\mathrm{IJ}]$ where $\mathrm{I} \in[\mathrm{CD}]$, $\mathrm{J} \in[\mathrm{AB}]$. We notice that $[\mathrm{IJ}]$ is a segment. According to the theorem 1 , the dual of $\mathrm{N}$ is a sinusoid curve between the duals of $I$ and $J$. As $I \in[C D], J \in[A B]$, the dual of $I$ is between the duals of the points $C$ and $D$. The dual of $J$ is also between the duals of the points $\mathrm{A}$ and $\mathrm{B}$. So, the dual of $\mathrm{N}$ is in the surfaces limited by the duals of $[\mathrm{AB}]$ and [CD]. Inversely, let $\mathrm{N}^{\prime}$ be a point in the surface limited by the dual of [AB] and [CD]. $\mathrm{N}^{\prime}$ is between two sinusoïd curves, the duals of two vertices $\mathrm{X}, \mathrm{Y}$ with $(\mathrm{X}, \mathrm{Y}) \in\{A, B, C, D\}^{2}$. A pixel is convex, so $[X Y] \subset$ p. According to the theorem 1 , there exists a point $\mathrm{s} \in[\mathrm{XY}]$ in the pixel such as dual(s) is a sinusoïd curve that pass through $\mathrm{N}^{\prime}$.

Secondly, one shows that the dual of a pixel is an area limited by the dual of its horizontal sides [AD] and [BC]. By analogy to the first case, we define a point $\mathrm{N}$ in a pixel where $\mathrm{N} \notin\{A, B, C, D\}$. There exists two points $\mathrm{I}$, $\mathrm{J}$ such that $\mathrm{N} \in$ $[\mathrm{IJ}]$ where $\mathrm{I} \in[\mathrm{AD}], \mathrm{J} \in[\mathrm{BC}]$. As $\mathrm{I} \in[\mathrm{AD}], \mathrm{J} \in[\mathrm{BC}]$, the dual of $\mathrm{I}$ is between the duals of $\mathrm{A}$ and $\mathrm{D}$. The dual of $\mathrm{J}$ is between the duals of $\mathrm{B}$ and $\mathrm{C}$. So, the dual of $\mathrm{N}$ is in the surfaces limited by the duals of $[\mathrm{AD}]$ and $[\mathrm{BC}]$. We can also notice that when we apply on the pixel p, a central symmetry by the center of the pixel, a horizontal side become a vertical side. Inversely, let $N^{\prime}$ be a point in the surface limited by the dual of [AD] and [BC]. N' is between two sinusoïd curves, the duals of two vertices $\mathrm{X}, \mathrm{Y}$ with $(\mathrm{X}, \mathrm{Y}) \in\{A, B, C, D\}^{2}$. A pixel is convex, so $[\mathrm{XY}] \subset \mathrm{p}$. According to the theorem 1 , there exists a point $\mathrm{s} \in[\mathrm{XY}]$ in the pixel such as dual(s) is a sinusoïd curve that pass through $\mathrm{N}$ '.

Thirdly, we show that the dual of a pixel is a surface limited by the diagonals segments [AC], [BD]. There exists two points $\mathrm{I}$, J such that $\mathrm{N} \in[\mathrm{IJ}]$ where $\mathrm{I} \in[\mathrm{AC}], \mathrm{J} \in[\mathrm{BD}]$. As $\mathrm{I} \in[\mathrm{AC}]$, $\mathrm{J} \in[\mathrm{BD}]$, the dual of $\mathrm{I}$ is between the duals of the points $A$ and $\mathrm{C}$. The dual of $\mathrm{J}$ is between the duals of the points $\mathrm{B}$ and $D$. So, the dual of $\mathrm{N}$ is in the surfaces limited by the duals of $[\mathrm{AC}]$ and [BD]. Inversely, let $\mathrm{N}^{\prime}$ be a point in the surface limited by the duals of [AC] and [BD]. $N^{\prime}$ is between two sinusoïd curves, the duals of two vertices $\mathrm{X}, \mathrm{Y}$ with $(\mathrm{X}, \mathrm{Y})$ $\in\{A, B, C, D\}^{2}$. A pixel is convex, so $[\mathrm{XY}] \subset \mathrm{p}$. According to the theorem 1 , there exists a point $s \in[X Y]$ in the pixel such as dual(s) is a sinusoïd curve which pass through N'.

We present an illustration of this proof in the following figure 10 where we can see the points $\mathrm{N}, \mathrm{I}, \mathrm{J}$ in the different cases of sides and diagonals : in 10a, we have the vertical sides; in $10 \mathrm{~b}$, the horizontal sides, and in 10c, the diagonals.

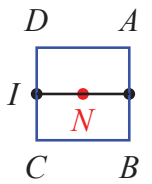

(a) $[\mathrm{AB}],[\mathrm{CD}]$

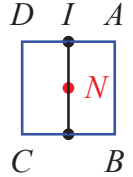

(b) $[\mathrm{AD}],[\mathrm{BC}]$

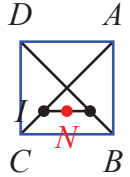

(c) $[\mathrm{AC}],[\mathrm{BD}]$
Figure 10: Segments of pixels

Let $\mathrm{p}$ a pixel centered in $(1,1)$ with the vertices $A, B, C, D$ as in the figure 10 . The following figures $11,12,13$ illustrate how we obtain the area of the dual of a pixel with the application of the previous theorem 2 : we compute the dual of sides and diagonals and we present their associated surface.

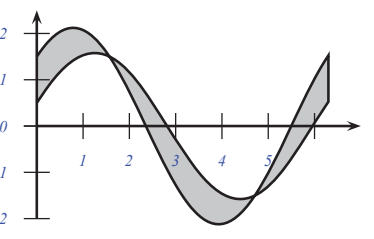

(a) Dual of $[\mathrm{AD}]$

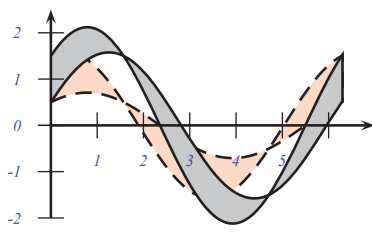

(c) Dual of $[\mathrm{AD}]$ and $[\mathrm{BC}]$

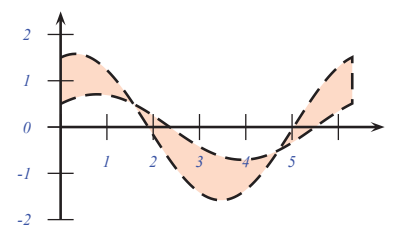

(b) Dual of $[\mathrm{BC}]$

(d) Surface

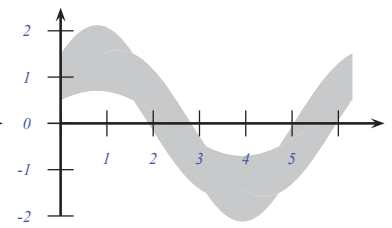

Figure 11: Horizontal sides 


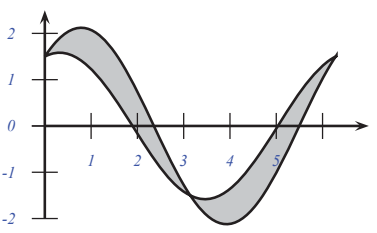

(a) Dual of $[\mathrm{AB}]$

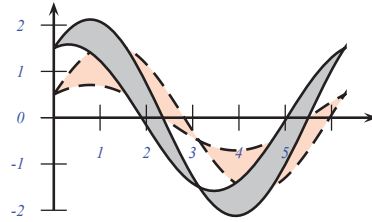

(c) Dual of $[\mathrm{AB}]$ and $[\mathrm{CD}]$

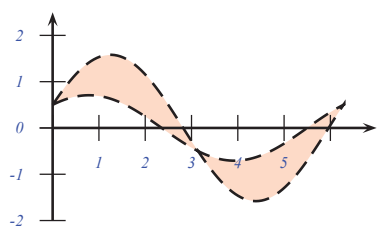

(b) Dual of [CD]
Figure 12: Vertical sides

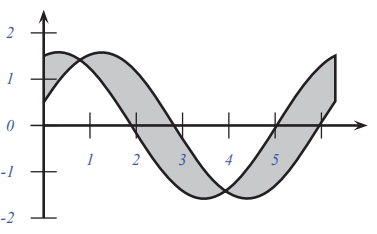

(a) Dual of the diagonal [BD]

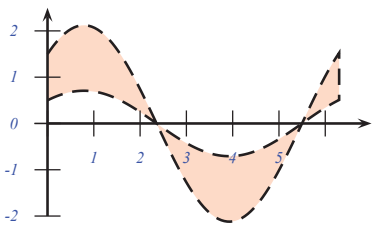

(b) Dual of the diagonal [AC]

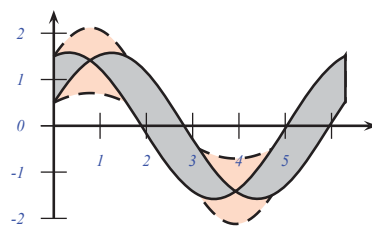

(c) Union of duals

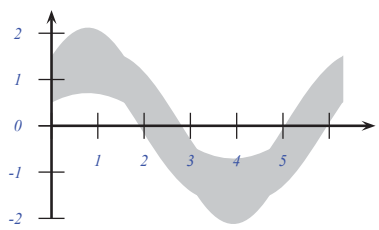

(d) Surface
Figure 13: Diagonals

We observe that in the previous figure 13, there does not exist empty space between the dual of the diagonals. Then, the dual of pixel is easy to be computed by the following theorem 3.

Theorem 3: the dual of a pixel is the union of the dual of its diagonal segments.

Proof: Let us prove that. Let $\mathrm{p}$ be a pixel with its vertices $\mathrm{A}, \mathrm{B}, \mathrm{C}, \mathrm{D}$ and its diagonals $[\mathrm{AC}]$ and $[\mathrm{BD}]$. In the previous theorem 2, we proved that the dual of a pixel is a surface between the dual of its diagonals. We are going to show that this area is equivalent to the union of the dual of its diagonals. That means, for a point in the dual of a pixel, it must belong to the dual of [AC] or the dual of [BD]. $[\mathrm{AC}]$ and $[\mathrm{BD}]$ are a common point, the center $\mathrm{O}$ of the pixel. So, the dual of $\mathrm{O}$ is a sinusoïd curve in the Dual([AC]) and the Dual([BD]) according to the theorem1. With $\theta \in[0$, $2 \pi]$, we can classify the $\operatorname{Dual}(\mathrm{A}), \operatorname{Dual}(\mathrm{B})$, Dual(O), Dual(C), $\operatorname{Dual}(\mathrm{D})$ by the relation $\leq$ or $\geq$. $\operatorname{Dual}(\mathrm{O})$ is in the middle of the classification.

Then, we have Dual $([\mathrm{AC}]) \subset \operatorname{Dual}([\mathrm{BD}])$ or Dual([BD]) $\subset$ Dual([AC])

As a conclusion, the set of points $\mathrm{N}$ verifying the proposition $[\mathrm{N} \in \operatorname{Dual}(\mathrm{p})$ with $\mathrm{N} \notin \operatorname{Dual}([\mathrm{AC}])$ and $\mathrm{N} \notin \operatorname{Dual}([\mathrm{BD}])]$ is $\phi$.

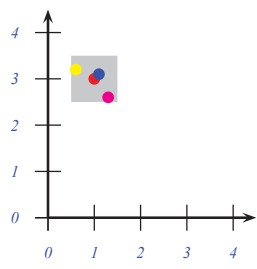

(a) Pixel $(1,3)$

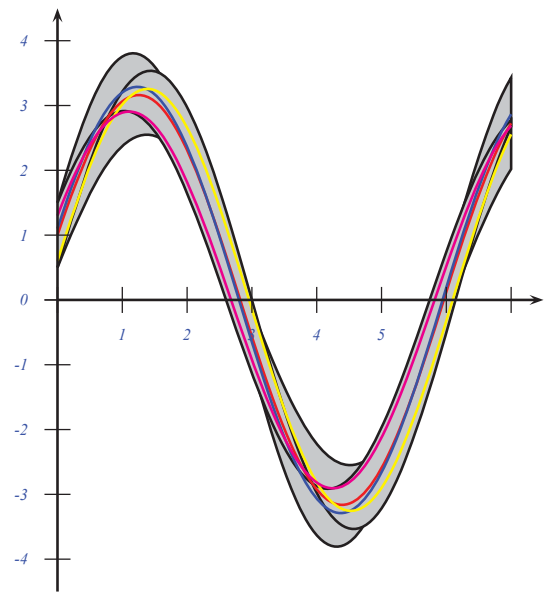

(b) Dual of the pixel $(1,3)$

Figure 14: Extended standard hough tranform

We see in the figure 14 , a pixel $(1,3)$ and its dual : we can notice that the dual of some points in the pixel is in the area.

1) Analysis of the dual: The purpose of this section is to analyze the dual of a pixel and to obtain some remarkable points that can be useful for an approximation of the dual.

Let $\mathrm{p}\left(p_{1}, p_{2}\right)$ be a pixel with its vertices: $\mathrm{A}\left(p_{1}+\frac{1}{2}, p_{2}+\frac{1}{2}\right)$, $\mathrm{B}\left(p_{1}+\frac{1}{2}, p_{2}-\frac{1}{2}\right), \mathrm{C}\left(p_{1}-\frac{1}{2}, p_{2}-\frac{1}{2}\right), \mathrm{D}\left(p_{1}-\frac{1}{2}, p_{2}+\frac{1}{2}\right)$.

The Dual(A), Dual (C), Dual(B), Dual(D) are respectively given by:

$$
\operatorname{Dual}(A): r_{A}=\left(p_{1}+\frac{1}{2}\right) \cos \theta+\left(p_{2}+\frac{1}{2}\right) \sin \theta
$$




$$
\begin{aligned}
& \operatorname{Dual}(C): r_{C}=\left(p_{1}-\frac{1}{2}\right) \cos \theta+\left(p_{2}-\frac{1}{2}\right) \sin \theta \\
& \operatorname{Dual}(B): r_{B}=\left(p_{1}+\frac{1}{2}\right) \cos \theta+\left(p_{2}-\frac{1}{2}\right) \sin \theta \\
& \operatorname{Dual}(D): r_{D}=\left(p_{1}-\frac{1}{2}\right) \cos \theta+\left(p_{2}+\frac{1}{2}\right) \sin \theta
\end{aligned}
$$

Considering (15), (17), (16), (18), with $\theta \in[0,2 \pi]$, we have the intersection points

$$
\begin{gathered}
\operatorname{Dual}(B) \cap \operatorname{Dual}(D)=, L \\
\operatorname{Dual}(A) \cap \operatorname{Dual}(C)=I, \\
\operatorname{Dual}(A) \cap \operatorname{Dual}(B)=O_{1}, \quad 0, \quad 1 \\
\operatorname{Dual}(A) \cap \operatorname{Dual}(D)=1, \quad 0 \\
\operatorname{Dual}(B) \cap \operatorname{Dual}(C)=0, \quad 1 \\
\operatorname{Dual}(C) \cap \operatorname{Dual}(D)=O_{0}, \quad 1, \quad 0
\end{gathered}
$$

The below table I gives more details on the coordinates of the points obtained in (19), (20), (21), (22), (23), (24).

Table I: Summary of intersections points

\begin{tabular}{|c|c|}
\hline$\theta$ & External points \\
\hline 0 & $O_{0}\left(0, p_{1}-\frac{1}{2}\right), O_{1}\left(0, p_{1}+\frac{1}{2}\right)$ \\
\hline$\frac{\pi}{2}$ & $0\left(\frac{\pi}{2}, p_{2}-\frac{1}{2}\right), 1\left(\frac{\pi}{2}, p_{2}+\frac{1}{2}\right)$ \\
\hline$\pi$ & $0\left(\pi,-\left(p_{1}+\frac{1}{2}\right)\right), \quad 1\left(\pi,-\left(p_{1}-\frac{1}{2}\right)\right)$ \\
\hline $3 \frac{\pi}{2}$ & $0\left(3 \frac{\pi}{2},-\left(p_{2}+\frac{1}{2}\right)\right), 1\left(3 \frac{\pi}{2},-\left(p_{2}-\frac{1}{2}\right)\right)$ \\
\hline $2 \pi$ & $0\left(2 \pi, p_{1}-\frac{1}{2}\right), \quad 1\left(2 \pi, p_{1}+\frac{1}{2}\right)$ \\
\hline & \\
\hline$\theta$ & Internal points \\
\hline$\frac{\pi}{4}$ & $\mathrm{~K}\left(\frac{\pi}{4},\left(p_{1}+p_{2}\right) \frac{\sqrt{2}}{2}\right)$ \\
\hline $3 \frac{\pi}{4}$ & $\mathrm{I}\left(\frac{3 \pi}{4}, \frac{\sqrt{2}}{2}\left(p_{2}-p_{1}\right)\right)$ \\
\hline $5 \frac{\pi}{4}$ & $\mathrm{~L}\left(\frac{5 \pi}{4},-\left(p_{1}+p_{2}\right) \frac{\sqrt{2}}{2}\right)$. \\
\hline $7 \frac{\pi}{4}$ & $\mathrm{~J}\left(\frac{7 \pi}{4}, \frac{\sqrt{2}}{2}\left(p_{1}-p_{2}\right)\right)$. \\
\hline
\end{tabular}

As we can see, in the figure 15, we have the points I, J of the intersection of the dual of A and C; in the figure 16, Those of the duals of $\mathrm{B}$ and $\mathrm{D}$ with the points $\mathrm{K}, \mathrm{L}$, the figure 17 show all the points.

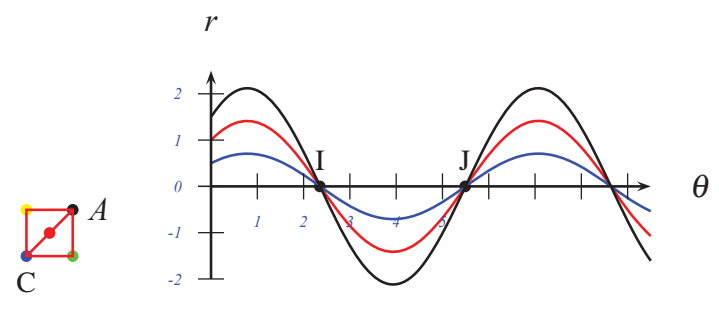
(a) $[\mathrm{AC}]$
(b) Dual of $[\mathrm{AC}]$

Figure 15: Dual of segment

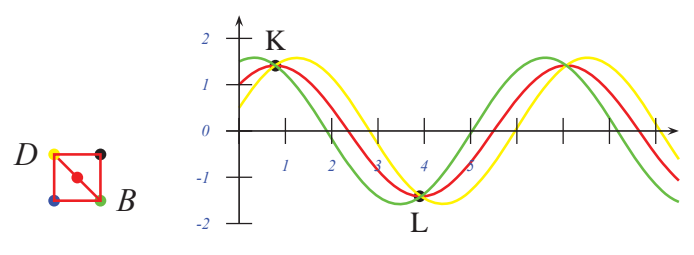

$\begin{array}{ll}\text { (a) }[\mathrm{BD}] & \text { (b) Dual of }[\mathrm{BD}]\end{array}$

Figure 16: Dual of segment

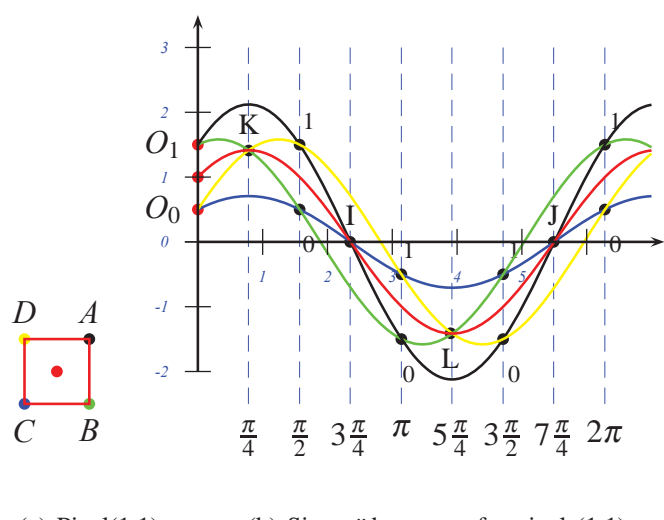

$\begin{array}{ll}\text { (a) Pixel }(1,1) & \text { (b) Sinusoïd curves of a pixel }(1,1)\end{array}$

Figure 17: Sinusoïd curves

When we follow the values of $\theta$ in [0 $2 \pi$ ], we will establish the following tables II, III, IV, V that compare in each interval, the values of the dual of vertices.

Table II: $\theta \in\left[0, \frac{\pi}{2}\right]$

\begin{tabular}{|c|c|c|}
\hline$\theta$ & $0 \leq \theta<\frac{\pi}{4}$ & $\frac{\pi}{4} \leq \theta<\frac{\pi}{2}$ \\
\hline relation & $r_{C} \leq r_{D} \leq r_{B} \leq r_{A}$ & $r_{C} \leq r_{B} \leq r_{D} \leq r_{A}$ \\
\hline
\end{tabular}

Table III: $\theta \in\left[\frac{\pi}{2}, \pi\right]$

\begin{tabular}{|c|c|c|}
\hline$\theta$ & $\frac{\pi}{2} \leq \theta<\frac{3 \pi}{4}$ & $\frac{3 \pi}{4} \leq \theta<\pi$ \\
\hline relation & $r_{B} \leq r_{C} \leq r_{A} \leq r_{D}$ & $r_{B} \leq r_{A} \leq r_{C} \leq r_{D}$ \\
\hline
\end{tabular}

Table IV: $\theta \in\left[\pi, \frac{3 \pi}{2}\right]$

\begin{tabular}{|c|c|c|}
\hline$\theta$ & $\pi \leq \theta<\frac{5 \pi}{4}$ & $\frac{5 \pi}{4} \leq \theta<\frac{3 \pi}{2}$ \\
\hline relation & $r_{A} \leq r_{B} \leq r_{D} \leq r_{C}$ & $r_{A} \leq r_{D} \leq r_{B} \leq r_{C}$ \\
\hline
\end{tabular}

Table V: $\theta \in\left[\frac{3 \pi}{2}, 2 \pi\right]$

\begin{tabular}{|c|c|c|}
\hline$\theta$ & $\frac{3 \pi}{2} \leq \theta<\frac{7 \pi}{4}$ & $\frac{7 \pi}{4} \leq \theta<2 \pi$ \\
\hline relation & $r_{D} \leq r_{A} \leq r_{C} \leq r_{B}$ & $r_{D} \leq r_{C} \leq r_{A} \leq r_{B}$ \\
\hline
\end{tabular}


When $\theta$ passes the values $\frac{\pi}{2}, \pi, 3 \frac{\pi}{2}, 2 \pi$, we will observe that the exteriors $r_{i}$ become the interiors $r_{i^{\prime}}$ and inversely.

According to

$$
\begin{array}{r}
r=x * \cos \theta+y * \sin \theta \Longleftrightarrow-r=x * \cos (\theta+\pi)+y * \\
\sin (\theta+\pi)
\end{array}
$$

we will work on an interval $[0, \pi]$, with the tables II, III and the external or internal points of this interval. The others exterior or interior points and the tables IV, $\mathrm{V}$ are deductions.

2) Approximation of the dual : Here, we propose an approximation of the area of the dual of a pixel. In computational geometry, algorithms exists to compute the intersection of convex polygons. A lot of methods of approximation possibility could be studied forward.

In our method, we consider a polygonal approximation based on a few points of the dual in the way to obtain some parameters $(\theta, \mathrm{r})$ : the idea is that if an object $\mathrm{O}^{\prime}$ is an approximation of an object $\mathrm{O}$ then $\operatorname{Dual}\left(\mathrm{O}^{\prime}\right)$ will be also an approximation of $\operatorname{Dual}(\mathrm{O})$.

Moreover, if $\mathrm{O}^{\prime} \subset \mathrm{O}$, we will have $\operatorname{Dual}\left(\mathrm{O}^{\prime}\right) \subset \operatorname{Dual}(\mathrm{O})$. Some points of the initial pixel are used to obtain its dual approximation.

Let $\mathrm{p}$ be a pixel centered in $\left(p_{1}, p_{2}\right)$. The pixel $\mathrm{p}$ has the vertices $\mathrm{A}\left(p_{1}+\frac{1}{2}, p_{2}+\frac{1}{2}\right), \mathrm{B}\left(p_{1}+\frac{1}{2}, p_{2}-\frac{1}{2}\right), \mathrm{C}\left(p_{1}-\frac{1}{2}\right.$, $\left.p_{2}-\frac{1}{2}\right), \mathrm{D}\left(p_{1}-\frac{1}{2}, p_{2}+\frac{1}{2}\right)$.

Let $p^{i}$ be a pixel centered in $\left(p_{1}, p_{2}\right)$ with its vertices $A^{i}\left(p_{1}+\right.$ $\left.\frac{1}{2}-i * \delta, p_{2}+\frac{1}{2}\right), B^{i}\left(p_{1}+\frac{1}{2}, p_{2}-\frac{1}{2}+i * \delta\right), C^{i}\left(p_{1}-\frac{1}{2}+i * \delta\right.$, $\left.p_{2}-\frac{1}{2}\right), D^{i}\left(p_{1}-\frac{1}{2}, p_{2}+\frac{1}{2}-i * \delta\right)$.

where $\mathrm{i} \in \mathbb{N}$ and $\delta \in[0,1]$ with the contraints $i * \delta \in\{0,1]$. The duals of the vertices of $p^{i}$ are :

$$
\begin{aligned}
& \operatorname{Dual}\left(A^{i}\right): r_{A^{i}}=\left(p_{1}+\frac{1}{2}-i * \delta\right) \cos \theta+\left(p_{2}+\frac{1}{2}\right) \sin \theta \\
& \operatorname{Dual}\left(C^{i}\right): r_{C^{i}}=\left(p_{1}-\frac{1}{2}+i * \delta\right) \cos \theta+\left(p_{2}-\frac{1}{2}\right) \sin \theta \\
& \operatorname{Dual}\left(B^{i}\right): r_{B^{i}}=\left(p_{1}+\frac{1}{2}\right) \cos \theta+\left(p_{2}-\frac{1}{2}+i * \delta\right) \sin \theta \\
& \operatorname{Dual}\left(D^{i}\right): r_{D^{i}}=\left(p_{1}-\frac{1}{2}\right) \cos \theta+\left(p_{2}+\frac{1}{2}-i * \delta\right) \sin \theta
\end{aligned}
$$

Then, $p^{i}$ is an approximative pixel of p where $i * \delta$ is the difference with the real vertice coordinates of $\mathrm{p}$. The maximal value of $i * \delta$ must be inferior to the maximal of the tolerance error.

We use the external points (see the table I) of the dual of the pixels $p^{0}, p^{1}, p^{2}, \ldots p^{n}$ to get a polygonal approximation of the dual of $\mathrm{p}$ with $p^{0}=p^{n+1}=p$.

We know that

$$
p^{n+1}=p \Longleftrightarrow(n+1) * \delta=1
$$

Then $(30) \Longleftrightarrow \delta=\frac{1}{n+1}$.

Finally, we need to take $\delta=\frac{1}{n+1}$ to have the pixels $p^{0}, p^{1}$, $p^{2}, \ldots, p^{n}$.

As we know, we work in $[0, \pi]$; we determine the approximation of the dual in this interval. We obtain the polygon
$\begin{array}{llllll}O_{1} & 1 & 1 & 0 & 0 & O_{0} \\ \end{array}$ details). The following figure 18 shows the polygon (in red color).

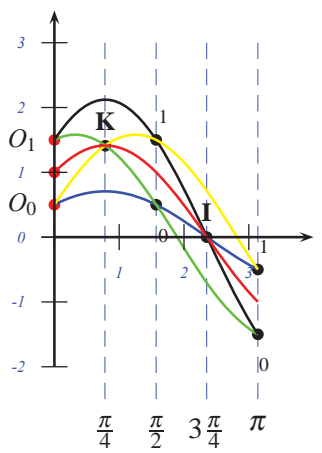

$\begin{array}{ll}\text { (a) Pixel }(1,1) & \text { (b) Sinusoïd curves of a pixel }(1,1)\end{array}$

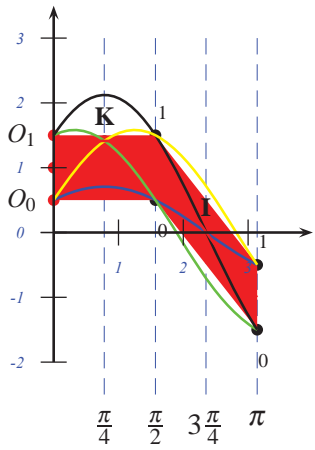

(c) Sinusoïd curves of $\mathrm{p}$

Figure 18: Approximative pixels Sinusoïd curves

The intersection points of vertices, with $\mathrm{i} \geq 1$ are $\operatorname{Dual}\left(A^{i}\right) \cap \operatorname{Dual}\left(B^{i}\right)=\left\{O_{1}^{i}\right\}, \quad \operatorname{Dual}\left(A^{i}\right) \cap \operatorname{Dual}\left(D^{i}\right)=\left\{\begin{array}{cc}i \\ 1\end{array}\right\}$, $\operatorname{Dual}\left(B^{i}\right) \cap \operatorname{Dual}\left(C^{i}\right)=\left\{\begin{array}{c}i \\ 0\end{array}\right\}, \operatorname{Dual}\left(C^{i}\right) \cap \operatorname{Dual}\left(D^{i}\right)=\left\{O_{0}^{i}\right\}$.

As $p^{i}$ is close of $\mathrm{p}$, these intersections points are close of $O_{1}, \quad 1, \quad 1, \quad 0, \quad 0, O_{0}$ respectively.

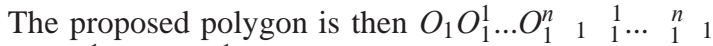

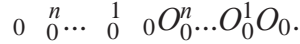

Let $S_{n}$ be the surface associated to this polygon. According to the tables II, III, we know that the surface of the dual of a pixel is :

$$
I=\int_{\left[0, \frac{\pi}{2}\right]}\left(r_{A}-r_{C}\right) d x+\int_{\left[\frac{\pi}{2}, \pi\right]}\left(r_{D}-r_{B}\right) d x=4
$$

According to (31), the extimated error is then $I-S_{n}=4-S_{n}$ and the percentage will be $\frac{4-S_{n}}{4}$.

If $\delta=\frac{1}{4}$, we will obtain $p^{1}=\mathrm{p}^{\prime}\left(p_{1}, p_{2}\right), p^{2}=\mathrm{p}$ ” $\left(p_{1}, p_{2}\right)$, $p^{3}=\mathrm{p}$ "' $\left(p_{1}, p_{2}\right)$ that are presented in the following figure 19 . 


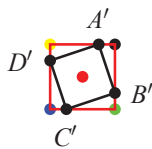

(a) pixel p'

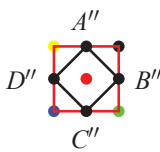

(b) pixel p"

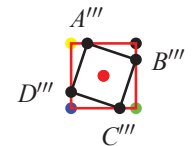

(c) pixel p"'
Figure 19: Approximative pixels

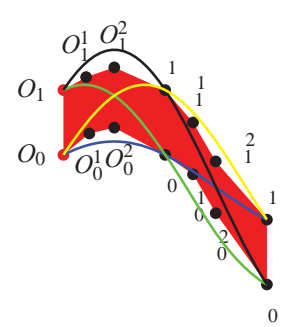

(a) $p^{2}$

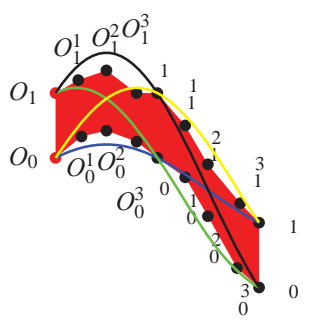

(b) $p^{3}$

Figure 21: Sinusoïd curves

\section{Preimage}

We present a new definition of preimage of pixels. Here, the term Dual means the Standard Hough transform. The preimage of $n$ pixels is an intersection of $n$ sinusoïd surfaces and differs from the definition introduced in[?], by Martine Dexet in her thesis.

Definition : Let $S=\left\{P_{1}, P_{2}, \ldots, P_{n-1}, P_{n}\right\}$ be a set of $n$ pixels $P_{i}$ in $\xi_{2}$. The preimage of $S$ is defined by $\operatorname{Preimage}(S)=\bigcap_{1 \leq i \leq n} \operatorname{Dual}\left(P_{i}\right)$.

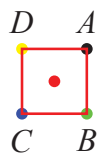

(a) Pixel(1,1)

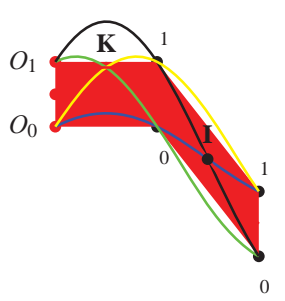

(b) $\mathrm{p}$

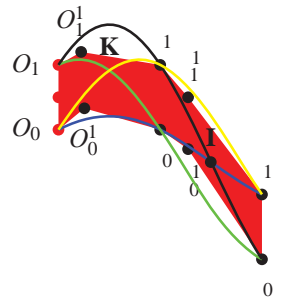

(c) $p^{1}$

Figure 20: Sinusoïd curves

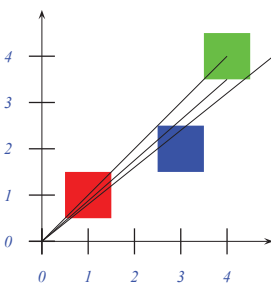

(a) pixels

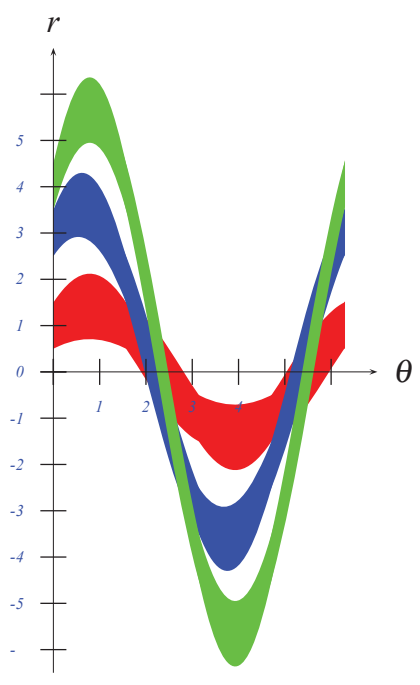

(b) Intersection of the Dual of pixels
Figure 22: Preimage of three pixels

In the figure 22, we see in 22a three colored pixels. In 22b, the corresponding colored dual of each pixel. The intersection of these three duals is a set of points representing the parameters $(\theta, r)$ of line that cross each pixel.

The parameters $(-r, \cos \theta, \sin \theta)$ are equal to the parameters $\left(c_{0}, c_{1}, c_{2}\right)$ of the analytical hyperplane in 2D dimension, 
presented in the section II-B. As you can see in the figure 22 the preimage of $n$ pixels give two areas because

$$
\begin{array}{r}
r=x * \cos \theta+y * \sin \theta \Longleftrightarrow-r=x * \cos (\theta+\pi)+y * \\
\sin (\theta+\pi)
\end{array}
$$

\section{Recognition algorithm}

In this part, we recall that the term dual indicates the Extended Standard Hough Transform. We can see that when, the dual of the losange of each pixel is considered, we will be in the case of naive line recognition.

Moreover, according to the definition of the naive and standard hyperplanes introduced in preliminaries and the geometry construction of these models, we see that the preimage contains the parameters of analytical line that crosses the pixels.

We propose the following algorithm :

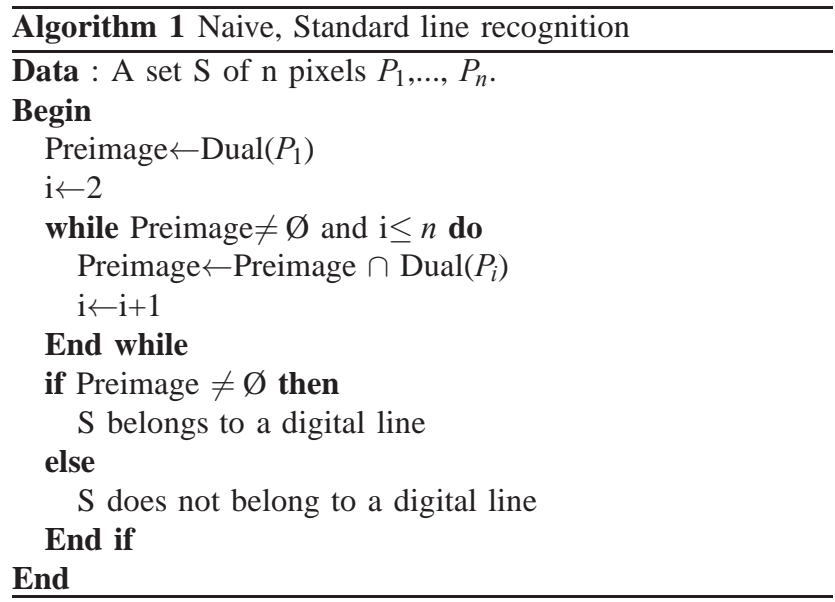

The Dual $\left(P_{i}\right)$ could be replaced by an approximation (polygon for example) in order to increase the performance of the algorithm.

The Extended Standard Hough Transform has its particularity to answer the question how can we recognize Naive and Standard by the standard Hough Transform. It conserves the properties of the Standard Hough Transform [6] as the size of the parameter space is limited $[6]: \theta \in[0, \pi]$ and $r$ $\in\left[0, \sqrt{c o l^{2}+r o w^{2}}\right\}$ with an image col x row. This leads to the vertical line detection.

\section{DUAL OF A TRIANGLE}

We are interested in how to compute the dual of a triangle. In case of triangle grid, we need to know how to determine the dual of a triangle. We establish the following theorem 4.

Theorem 4: The dual of a triangle is the union of the dual of one of its two adjacent(consecutif) sides

Proof: Let [AC] and $[\mathrm{AB}]$ be two adjacents sides of a triangle $(\mathrm{ABC})$ as we see in the following figure 23 . Let $\mathrm{N}$ be a point in the triangle (if $\mathrm{N}$ is a vertex the proof is realized). There exists two point $I$ and $J$ such that $I \in[A C], J \in[A B]$ and $N \in[I J]$. By analogy with the proof of the theorem 2. We have two consecutif sides $[A C]$ and $[A B]$ that are secants on the vertex $A$ of a triangle $(A B C)$. Then, the surface between the dual of $[\mathrm{AC}]$ and $[\mathrm{AB}]$ does not contains empty space. Morover, we knows that the dual of $\mathrm{I}$ is between the dual of $\mathrm{A}$ and $\mathrm{C}$ like the dual of $\mathrm{J}$ is between the dual of $\mathrm{A}$ and $\mathrm{B}$. As we know the dual of $\mathrm{N}$ is between the dual of $\mathrm{I}$ and the dual of $\mathrm{J}$, then the dual of $\mathrm{N}$ is in the union of the dual of $[\mathrm{AC}]$ and $[\mathrm{AB}]$.

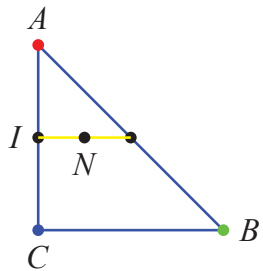

Figure 23: Triangle

The below figure 24 shows the dual of a triangle, it contains the dual of each point of the triangle : In 24b, the dual of the vertex of a triangle in $24 \mathrm{a}$, and in $24 \mathrm{c}$, the dual of a triangle.

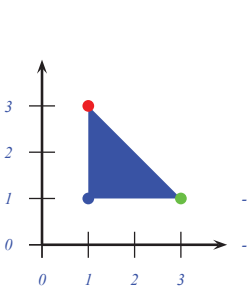

(a) Triangle

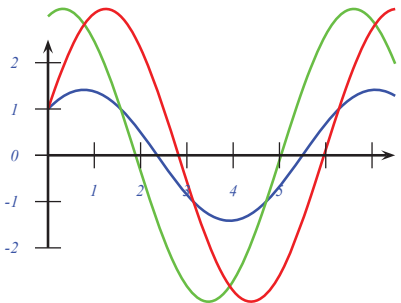

(b) Dual of segments

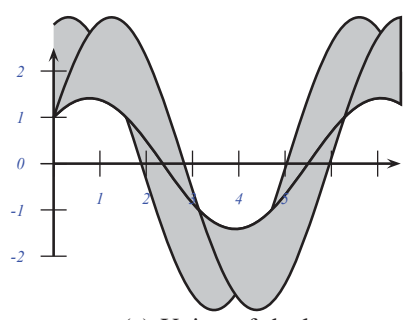

(c) Union of duals

Figure 24: Extended standard hough transform

\section{Conclusions}

This paper establishs a new method based on the Standard Hough Transform for the recognition of naive or standard lines in a noisy picture. We introduced a new definition of preimage that allows us to obtain an algorithm recognition. We obtained that the dual of a pixel is an non polygonal area. That leads 
to propose an approximation. Others approximative methods could be studied deeply forward in the objectif to improve the algorithm. Some works still left to be done, particularly the implemention on concrete pictures. The dual of a triangle has been proposed. That could give interested perpectives in the others grids. One of the remaining question is to extend the method in 3D.

\section{ACKNOWLEGDMENT}

The authors would like to thank the organization “L'Agence Universitaire de la Francophonie"(AUF) for their help and support in making this work possible.

\section{REFERENCES}

[1] E. Andres. Discrete linear objects in dimension $\mathrm{n}$ : the standard model. Graphical Models, pages 65 :92-111, 2003.

[2] E. Andres, R. Acharya, C. Sibata, Discrete analytical hyperplanes, Graphical Model and Image processing, Volume 59, Issue 5, September 1997, Pages 302-309.

[3] D.H.Ballard. Generalizing the hough transform to detect arbitrary shapes. Pattern Recognition, Vol. 13, No. 2, pp. 111-112, 1981.

[4] M Bruckstein, N.Kiryati, Y.Eldar. A probabilistic hough transform. Pattern Recognition, 24(4) : 303-316, 1991.

[5] M.Dexet. Architecture d'un modéleur géometrique à base topologique d'objets discrets et methodes de reconstruction en dimensions 2 et 3 . Thèse de Doctorat, Université de Poitiers, France, 2006.

[6] F. Dubeau, S.El mejdani, R.Egli. Champs de hauteur de la transformée de hough standard. Actes de 4ième Conférence Internationale en Recherche Opérationnelle, pages 133-144, 2005.

[7] J. Cha , R. H. Cofer , S. P. Kozaitis, Extended Hough transform for linear feature detection, Pattern Recognition, v.39 n.6, p.1034-1043, June, 2006

[8] H.Maître. Un panorama de la transformée de hough - a review on hough transform. Traitement du Signal, 2(4) :305-317, 1985.

[9] J. Matas, C. Galambosy and J. Kittler. Progressive probabilistic hough transform, Transform, Volume: 24, n.4, Pages: 303-316, 1991

[10] J. P Reveillès, combinatorial pieces in digital lines and planes, in Vision Geometry IV Proc. SPIE, Vol. 2573, San Diego, CA, 1995, pp. 23-24.

[11] J.P. Reveillès. Géométrie discrète, Calcul en nombres entiers et algorithmiques. Thèse d'état, Université Louis Pasteur, Strasbourg, France, 1991 